\title{
Rotierender optischer Positions- und Winkelsensor
}

\author{
M. Bibl, R. Stein, E. Döberl, G. Schitter
}

In dieser Arbeit wird ein Sensorprinzip vorgestellt, welches die Höhe und den Winkel des Referenzpunkts des Sensors in Bezug auf eine Laserreferenz optisch messen kann. Dies wird durch eine Zeitmessung zwischen den Laserimpulsen und einem Hallimpuls als Referenz erreicht. Es werden Hard- und Softwaremaßnahmen zur Erkennung und Unterdrückung von Störungen vorgestellt und implementiert. Mit dem Sensorprinzip kann eine Standardabweichung von 0,131 mm bei einem Messbereich von $\pm 92 \mathrm{~mm}$ erreicht werden, wobei sich die Mittelwerte der unterschiedlichen Messmethoden um max. 0,393 mm unterscheiden. Bei geringer Anforderung an die Messfrequenz des Sensors können diese Werte durch Mittelwertbildung noch weiter verbessert werden.

Schlüsselwörter: Positions- und Winkelmessung; rotierender optischer Sensor; optische Messtechnik

\section{Rotating optical height and angle sensor.}

This work presents a sensor which is able to measure the height and the angle of the reference point of the sensor in respect to a laser reference. This is done by measuring the time between laser pulses and a hall sensor as a reference. Also, the implementation of the measurement failure handling in hardware and software is discussed. With the presented sensor a standard deviation of $0.131 \mathrm{~mm}$ at a measurement range of $\pm 92 \mathrm{~mm}$ can be achieved. The maximum deviation of the mean values of the different measurement methods is $0.393 \mathrm{~mm}$. If there is no need for a high bandwidth of the sensor, these values can be further decreased by calculating average values of the measurement signal.

Keywords: height and angle measurement; rotating optical sensor; optical metrology

Eingegangen am 22. Mai 2018, angenommen am 5. September 2018, online publiziert am 18. September 2018

(c) The Author(s) 2018

\section{Einleitung}

Beim Bau moderner Gebäude wird immer mehr Wert auf Energieeffizienz [1] und Wohlbefinden [2] im Gebäude gelegt. Um das Ansprechverhalten der Fußbodenheizung und damit auch die Temperaturstabilität im Raum zu verbessern, wird versucht die Estrichschicht so dünn wie möglich auszuführen. Dafür sind jedoch festere Estriche notwendig, die schwieriger zu verarbeiten sind [3], weshalb die Nivellierung und Glättung des Estrichs automatisiert werden soll. Um diese Arbeitsschritte zu automatisieren muss die Position des Nivellierwerkzeugs gemessen werden. Für die Aufgabe der optischen Entfernungsmessung finden sich eine Fülle an Verfahren in der Literatur wie z.B. Interferometrie, Laserdistanzmessung, Lasertriangulation oder konfokale Abstandsmessung [4] welche den Abstand eines Punkts vom Sensor ermitteln.

Auf heutigen Baustellen wird meist am Eingang ein sogenannter "Meterriss"einen Meter über dem fertigen Fußboden angebracht und auf diesen Meterriss werden dann alle Maße bezogen. Üblicherweise wird anschließend ein Rotationslaser aufgestellt, der diesen Meterriss im ganzen Raum millimetergenau weitergibt [5] und auf den sich dann alle Maße beziehen. Als Empfängerprinzipien für diese Laser Referenz sind z.B. der Vier-Quadranten Detektor [6] oder die laterale Photodiode [6] bekannt. Aufgrund der Verwendung eines Rotationslasers sind die im vorigen Absatz genannten Messmethoden ungeeignet, da diese den Abstand nicht im Bezug auf den Rotationslaser bestimmen können. Als Empfänger des Rotationslasers wird aktuell z.B. der Hilti PRA 38 verwendet, mit einer Genauigkeit von $\pm 0,5 \mathrm{~mm}$ bei einem Messabstand bis zu $10 \mathrm{~m}$ [7]

In dieser Arbeit wird ein Sensor vorgestellt, welcher die Höhenverschiebung und die Rotation des Sensors im Vergleich zur zuvor beschriebenen Laserreferenz messen kann, wobei die Erfassung des Laserstrahls mit Photodioden realisiert wird. Mit diesem Sensor können anschließend automatisierte Verlegesysteme ausgestattet werden, um die Verlegung des Estrichs und der Dämmschicht darunter zu automatisieren.

\section{Aufbau und Funktion des Sensors}

In diesem Abschnitt werden der Aufbau des Sensors und die Algorithmik zur Ermittlung der Messwerte präsentiert.

\subsection{Mechanischer Aufbau}

Der entwickelte Sensor ist wie in Abb. 1 dargestellt aufgebaut. Er besteht aus einem feststehenden Gehäuse (Stator) und einem rotierenden inneren Teil (Rotor), welcher über einen Gleichstrommotor angetrieben wird. Der Stator dient der Montierung des Sensors und trägt einen Permanentmagneten. Der Rotor trägt zwei Photodioden, welche das Auftreffen des Laserstrahls registrieren und dabei einen elektrischen Strom generieren. Dieser Strom wird an einem Digitaleingang eines Mikrocontrollers, welcher auf dem Rotor sitzt, detektiert. Wird der Winkel des Rotors in Bezug zum Stator beim Auftreffen des Lasers für beide Photodioden gemessen (siehe Abb. 2

Bibl, Matthias, Institut für Automatisierungs- und Regelungstechnik (ACIN), Technische Universität Wien, Gußhausstraße 27-29, 1040 Wien, Österreich

(E-Mail: matthias.bibl@tuwien.ac.at); Stein, Robert, ASA Astrosysteme GmbH, Galgenau 19, 4212 Neumarkt im Mühlkreis, Österreich; Döberl, Egon, ASA Astrosysteme GmbH, Galgenau 19, 4212 Neumarkt im Mühlkreis, Österreich; Schitter, Georg, Institut für Automatisierungs- und Regelungstechnik (ACIN), Technische Universität Wien, Gußhausstraße 27-29, 1040 Wien, Österreich 


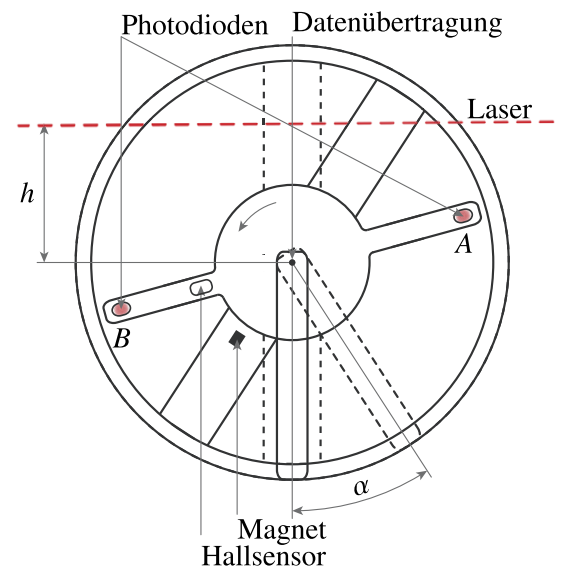

Abb. 1. Aufbau des Lasersensors mit zwei Photodioden und einem Hallsensor zur Nulldurchgangsdetektion. Die strichlierte Darstellung zeigt den feststehenden Teil des Sensors bei einer Rotation um den Winkel $\alpha$. Die Höhe $h$ und der Winkel $\alpha$ sollen gemessen werden. Die Laserlinie wird von einem externen Laser erzeugt

Winkel $\gamma$ und $\delta$ ), lässt sich daraus die zu ermittelnde Höhe $h$ und der Drehwinkel $\alpha$ berechnen. Die Winkel $\gamma$ und $\delta$ werden von einem Bezugspunkt aus gemessen. Der Bezugspunkt wird durch einen Hallsensor erfasst, wobei als $0^{\circ}$ jener Punkt festgelegt wird, an dem der Hallsensor den Magneten am Stator passiert. Die aus der Messung ermittelten Werte $h$ und $\alpha$ werden optisch mit einer RS-232 Schnittstelle, welche im Drehpunkt des Rotors sitzt, übertragen. Die Höhe $h$ wird in Bezug zum Drehpunkt des Rotors gemessen, wobei der Drehpunkt des Rotors $0 \mathrm{~mm}$ entspricht.

\subsection{Messung der Winkel}

Um die Winkel beim Auftreffen des Laserstrahls auf die Photodioden in Bezug zum Nullpunkt zu bestimmen, wurde anfangs ein inkremental Encoder im Antriebsmotor verwendet. Dies bringt den Vorteil, dass der Winkel direkt ausgelesen werden kann und automatisch auf den Nullpunkt des inkremental Encoders bezogen ist. Die Montage eines inkremental Encoders mit entsprechender Auflösung ist jedoch mechanisch schwierig, deswegen wurde eine andere Messmethode entwickelt. Es wird beim Nulldurchgang (Impuls des Hallsensors) ein Timer des verwendeten Microcontrollers gestartet. Wenn der Laserstrahl auf eine Photodiode trifft, wird der Timerwert zu diesem Zeitpunkt gespeichert. Wenn die Dauer einer Umdrehung bekannt ist, welche der Dauer zwischen zwei Hallimpulsen entspricht, kann der Winkel beim Auftreffen des Laserstrahls bestimmt werden. Es folgt der Zusammenhang

$$
\delta=\frac{Z}{D} 360
$$

für den Winkel in ${ }^{\circ}$, wobei $Z$ den Zählerwert beim Auftreffen des Laserstrahls und $D$ die Dauer einer Umdrehung in Zählerticks darstellt.

\subsection{Berechnung der Höhe und des Winkels}

Jede Photodiode schneidet pro Umdrehung zwei Mal den Laserstrahl. Die Photodioden werden mit Buchstaben versehen ( $A$ und $B$ ) und das Schneiden mit der Laserlinie erhöht den Index der jeweiligen Photodiode $\left(A_{1}, B_{1}\right.$, usw.), wie in Abb. 2 dargestellt. Mit den beiden Winkeln $\delta$ und $\gamma$ kann die Höhe $h$ und der Winkel $\alpha$ berechnet werden. Es folgt der Zusammenhang

$$
h_{\mathrm{AB}}=r \sin \left(\frac{B_{1}-A_{1}}{D} \pi\right) \text { und }
$$

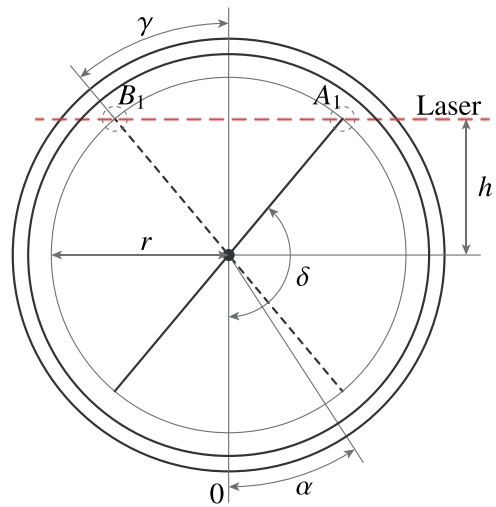

Abb. 2. Schematische Darstellung des Sensors zur Verdeutlichung der Zusammenhänge bei der Berechnung der Höhe $\boldsymbol{h}$ und des Winkels $\boldsymbol{\alpha}$ aus den gemessenen Winkelwerten $\gamma$ und $\delta$

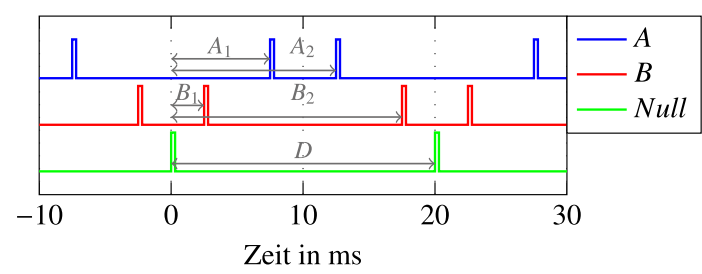

Abb. 3. Timing Diagramm des Sensors für eine ganze Umdrehung (Bereich zwischen 0 und $20 \mathrm{~ms}$ ). Es sind die Signale der Photodiode $A$ (Blau), B (Rot) und der Index (Grün) zur Nulldurchgangsdetektion dargestellt. Die Höhe der Laserlinie beträgt $65,054 \mathrm{~mm}$ bei einem Messbereich von $92 \mathrm{~mm}$ und der Drehwinkel $\alpha$ beträgt $0^{\circ}$. Die Drehzahl des Rotors beträgt $3000 \mathrm{~min}^{-1}$ (Farbabbildung online)

$$
\alpha_{\mathrm{AB}}=180 \frac{B_{1}+A_{1}}{D},
$$

wobei $r$ den Radius vom Mittelpunkt des Sensors zur Position der Photodiode darstellt. Der Zusammenhang aus Formel (2) und (3) gilt auch für die Berechnung mit den Werten $A_{2}$ und $B_{2}$.

Die Berechnung von $h$ und $\alpha$ kann auch mit den Werten von $A_{1}$ und $A_{2}$ durchgeführt werden. Dadurch kann die Anzahl der Messwerte pro Umdrehung verdoppelt werden und es folgt der Zusammenhang

$$
\begin{aligned}
h_{\mathrm{AA}} & =r \cos \left(\frac{A_{2}-A_{1}}{D} \pi\right) \text { und } \\
\alpha_{\mathrm{AA}} & =180 \frac{A_{1}+A_{2}}{D} .
\end{aligned}
$$

In Abb. 3 ist die Impulsabfolge für eine Höhe des Lasers von 65,054 $\mathrm{mm}$ bei einem Messbereich von $92 \mathrm{~mm}$ und einem Winkel $\alpha=0^{\circ}$ dargestellt. Die Drehzahl des Rotors beträgt $3000 \mathrm{~min}^{-1}$. Nimmt die Höhe des Laserstrahls zu, nimmt der Abstand zwischen $B_{1}$ und $A_{1}$ zu und die berechnete Höhe nimmt ebenfalls zu. Dies geschieht bis $B_{1}=0$ und $A_{1}=D / 2$, dann ist das Ende des Messbereichs erreicht, welcher dem Radius $r$ des Montierungskreises der Photodioden entspricht. Bei abnehmender Höhe des Laserstrahls, nimmt der Abstand zwischen $B_{1}$ und $A_{1}$ ab, bis diese gleich großsind. Dann ist der Nullpunkt der Messung erreicht. Bei noch weiterer Reduktion der Höhe des Lasers in den negativen Messbereich tritt $A_{1}$ vor $B_{1}$ auf und die beiden Signale tauschen die Rolle aus der Überlegung für den positiven Messbereich. 


\subsection{Erhöhung der Messrate}

Der Sensor aus Abb. 1 ist mit zwei Photodioden ausgestattet, welche pro Umdrehung jeweils zwei Mal den Laserpfad schneiden. Wird die Anzahl der Laserdioden verdoppelt indem ein zusätzlicher "Arm" am Rotor hinzugefügt wird, kann die Messfrequenz ebenso verdoppelt werden. Ein Sensor mit vier Photodioden, welcher für die Messungen verwendet wurde, ist in Abb. 4 dargestellt. Es können pro Umdrehung acht Messwerte gewonnen werden, wobei der Winkel nur im Bereich $\pm 90^{\circ}$ gemessen werden kann. Die Photodioden werden mit $A, B, C$ und $D$ bezeichnet. Die ermittelten Höhen werden in Folge mit $h_{A 2 A 1}=h_{1}, h_{C 2 A 2}=h_{2}, h_{C 2 C 1}=h_{3}, h_{A 1 C 1}=h_{4}, h_{B 2 B 1}=h_{5}$, $h_{B 2 D 2}=h_{6}, h_{D 2 D 1}=h_{7}, h_{B 1 D 1}=h_{8}$ bezeichnet.

\subsection{Störunterdrückung}

Das Lasersignal, das der Sensor empfängt, wird mittels eines $360^{\circ}$ Lasers (PLS360E, PLS, Berg, Deutschland) erzeugt. Dieses Lasersignal wird mit einem Rechtecksignal mit einer Frequenz von $36 \mathrm{kHz}$ und einem Puls- Pausenverhältnis von 80 zu 20 moduliert um eine höhere Signalleistung am Demodulator zu erreichen. Das Signal der Photodioden wird mittels einem Demodulator (VSOP58416, Vishay Intertechnology, Inc., Malvern, USA) demoduliert, welcher für das Demodulieren von Infrarot Fernbedienungssignalen ausgelegt ist [8]. Wenn der Baustein das Pulsmuster mit der richtigen Frequenz erkennt, wird der Ausgang aktiviert solange das Lasersignal anliegt. Somit kann zwischen Fremdlicht (Umgebungslicht, andere Laserquellen, usw.) und der Laserreferenz unterschieden werden. Sollte die Störlichtintensität sehr hoch werden (z.B.: direkte Sonneneinstrahlung auf die Photodioden), gelangen die Photodioden in Sättigung und es kann durch die hohe Gleichspannungsaussteuerung der Photodiode kein Wechselsignal mehr empfangen werden [9]. In diesem Fall ist keine Messung mehr möglich. Der Sensor erkennt, dass keine Signale mehr empfangen werden und signalisiert dies durch eine rote LED.

Bei einer statischen Höhe des Lasers und des Sensors und einer konstanten Drehzahl des Rotors ist die Abfolge der Winkel bekannt. Es wurde eine Logik implementiert, die jeden empfangenen Laserimpuls auf Gültigkeit überprüft. Sollte ein Laserimpuls nicht in der vorhergesehenen Reihenfolge auftreten, werden die fehlerhaft gemessenen Winkel verworfen und die Messung wird mit den nachfolgend aufgenommenen Winkeln fortgesetzt. Dies kann durch eine externe Störung, beispielsweise durch eine Infrarot Fernbedienung, oder durch Reflexionen des Lasers und einer dadurch verursachten verzögerten doppelten Erkennung des Laserimpulses auftreten.

\subsection{Zeitmessung der Laserimpulse}

Wie in Abschn. 2.2 beschrieben, werden die Winkel beim Laserkontakt über eine Zeit gemessen. Um diese Zeit möglichst genau zu Messen, da die Zeitauflösung direkt die Winkelauflösung beeinflusst, werden zwei Timer des verwendeten Mikrocontrollers (STM32F103CBT6, STMicroelectronics, Amsterdam, Niederlande) verwendet. Diese beiden Timer werden gleichzeitig gestartet und zählen mit einer Taktfrequenz von 1,2 MHz. Der erste Timer bestimmt die Periodendauer der Rotorumdrehung, welche im Bereich von 20 ms liegt. Bei einem Impuls des Hallsensors wird ein Interrupt generiert und die verstrichene Zeit seit dem letztem Interrupt ermittelt. Der zweite Timer misst die Auftrittszeit der Laserimpulse an den Photodioden. Beide Timer werden im Input Capture Mode betrieben [10], bei dem bei Auftreten des Interrupts am Eingang der Timerwert in ein eigenes Register gesichert wird. Somit ist die Erfassung des Zeitpunkts des Laserimpulses aufgrund der Verzögerung des Mikrocontrollers auf einen Zeitschritt (833,3 ns) genau. Mit dem Radius $r$ des Rotors von 92 mm und einer Drehzahl des Rotors von

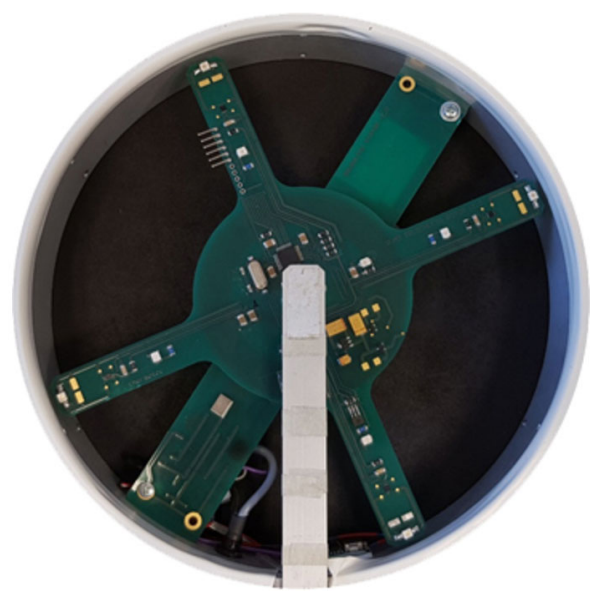

Abb. 4. Aufbau des Lasersensors, der für die Messungen verwendet wurde. Die Energieübertragung vom Stator zum Rotor erfolgt induktiv über zwei Spulen, die sich auf den Platinen befinden. Am Stator wird ein magnetisches Wechselfeld erzeugt, welches in der Spule im Rotor einen elektrischen Wechselstrom hervorruft. Dieser Wechselstrom wird gleichgerichtet und mittels Stützkondensatoren in eine Gleichspannung gewandelt

$3000 \mathrm{~min}^{-1}$ ergibt sich eine Winkelungenauigkeit von $0,015^{\circ}$. Dies entspricht einer max. Ungenauigkeit für die gegebene Sensorgeometrie bei der Höhenmessung von $12 \mu \mathrm{m}$.

\section{Messungen}

Um die Funktion des Sensors zu überprüfen wurde der Sensor aus Abb. 4 verwendet. Laser und Sensor bleiben bei der Messung sowohl in der Höhe als auch im Winkel konstant und es werden keine zusätzlichen Störungen eingebracht. Der Raum ist ein Laborraum mit normalen Tageslichtumgebungen, jedoch ohne direkter Sonneneinstrahlung auf die Detektoren. Die Messdauer beträgt 100 s, wobei die beschriebene Achtfachauswertung sowohl für die Höhe als auch für den Winkel verwendet wird. Die Ergebnisse sind in Abb. 5 dargestellt. Die roten Linien stellen den Mittelwert und die grünen Linien die Standardabweichung der jeweiligen Messmethode dar. Um die Grafik übersichtlich zu halten, werden nur die ersten $10 \mathrm{~s}$ der Messung gezeigt. In Abb. 6 sind die Verteilungsdichtefunktionen der Zeitsignale aus Abb. 5 in blau gezeigt. Es wurde die gesamte Signallänge von $100 \mathrm{~s}$ für die Berechnung verwendet. Die aus den Daten errechnete Normalverteilung ist jeweils in rot dargestellt. Aus den Abbildungen für die Messmethoden $h_{1}$ bis $h_{8}$ kann geschlossen werden, dass die Messfehler normalverteilt sind und die Annahme einer Normalverteilung zulässig ist. In Tab. 1 sind die Mittelwerte und Standardabweichungen aus Abb. 6 aufgelistet. Bei der angestrebten Anwendung des Sensors als Höhenmessung zur automatischen Verlegung von Dämmschüttungen und Estrichen ist die Messfrequenz von $400 \mathrm{~Hz}$ nicht notwendig, da erwartet wird, dass sich die zu messende Höhe nur langsam ändert. Es kann daher über mehrere Messwerte ein gleitender Mittelwert gebildet werden. Wird eine Normalverteilung für die Fehler im Messsignal angenommen, verringert sich die Standardabweichung um die Wurzel aus der Anzahl der Messwerte die zur Mittelwertbildung herangezogen werden [11]. Wird der gleitende Mittelwert aus vier Messwerten berechnet, verringert sich die Messfrequenz des Sensors, jedoch halbiert sich dadurch auch die Standardabweichung, womit eine Standardabweichung von $66 \mu \mathrm{m}$ erreicht werden kann.

Um die Abweichung des Sensors für die Messmethoden $h_{1}$ bis $h_{8}$ über den gesamten Messbereich zu ermitteln, wurde der Sensor 


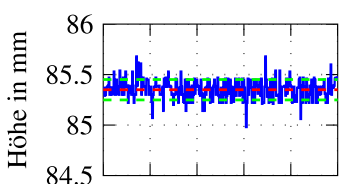

(a) $h_{1}$

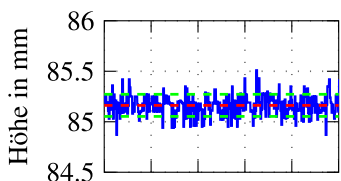

(c) $h_{3}$

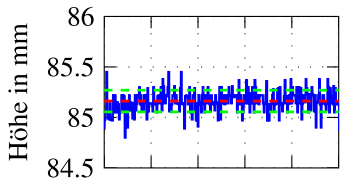

(e) $h_{5}$

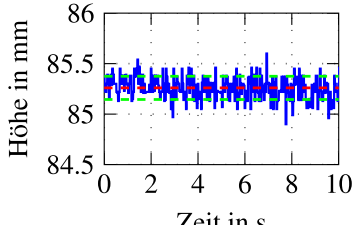

(g) $h_{7}$

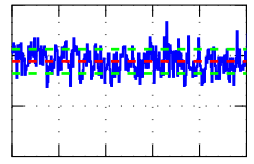

(b) $h_{2}$

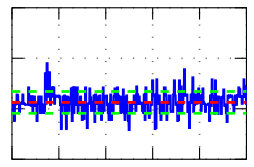

(d) $h_{4}$

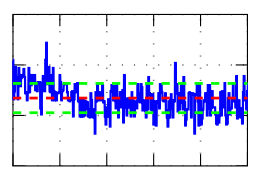

(f) $h_{6}$

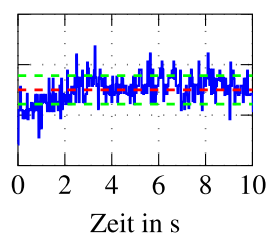

(h) $h_{8}$
Abb. 5. Ermittelte Höhenwerte der acht unterschiedlichen Messungen (a) $h_{1}$ bis (h) $h_{\mathbf{8}}$. Die Mittelwerte sind rot strichliert und der Mittelwert \pm der Standardabweichung ist grün strichliert dargestellt. Bei dieser Messung werden der Sensor und der Laser auf einer Höhe fixiert und keine Mittelwerte berechnet. Die Messdauer beträgt $100 \mathrm{~s}$, zur besseren Lesbarkeit werden nur die ersten $10 \mathrm{~s}$ dargestellt (Farbabbildung online)

Tab. 1. Mittelwerte und Standardabweichungen der Messwerte aus Abb. 6

\begin{tabular}{lll}
\hline Messmethode & Mittelwert in mm & Standardabweichung in mm \\
\hline$h_{1}$ & 85,398 & 0,103 \\
$h_{2}$ & 85,499 & 0,129 \\
$h_{3}$ & 85,215 & 0,121 \\
$h_{4}$ & 85,106 & 0,112 \\
$h_{5}$ & 85,187 & 0,112 \\
$h_{6}$ & 85,234 & 0,137 \\
$h_{7}$ & 85,299 & 0,110 \\
$h_{8}$ & 85,251 & 0,131 \\
\hline
\end{tabular}

auf eine Linearachse (DGE-18-80-ZR-LV-RK-KF-GK, Festo AG \& Co. $K G$, Esslingen am Neckar, Deutschland) montiert, welche von einem Motor (AM8023-0E21-0000, Beckhoff Automation GmbH \& Co. $K G$, Verl, Deutschland) angetrieben wird. Die Positionsermittlung der Achse erfolgt durch den im Motor verbauten inkremental Encoder, wobei ein Inkrement 50 nm entspricht. Der Laser wird auf eine konstante Höhe eingestellt und der Sensor mittels der Linearachse so eingerichtet, dass der Messwert $h 0 \mathrm{~mm}$ ergibt. Anschließend werden über die Echtzeit SPS TwinCAT Positionen im Bereich $-80 \mathrm{~mm}$ bis $+80 \mathrm{~mm}$ in $5 \mathrm{~mm}$ Schritten angefahren, die Höhenwerte $h_{1}$ bis $h_{8}$ gemessen und die Abweichung zum Sollwert bestimmt. Das Ergebnis der Messung ist in Abb. 7 dargestellt. Es ist zu sehen, dass der absolute Messfehler über den gesamten Messbereich für jede

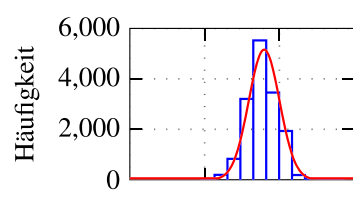

(a) $h_{1}$

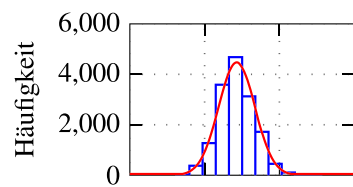

(c) $h_{3}$

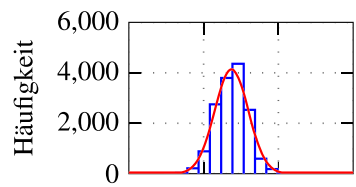

(e) $h_{5}$

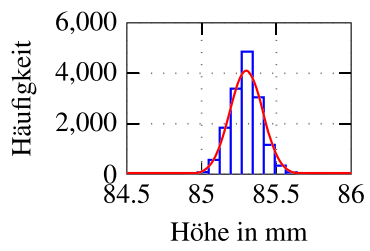

(g) $h_{7}$

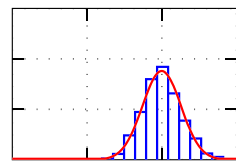

(b) $h_{2}$

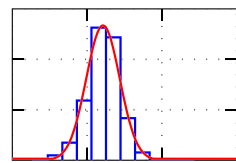

(d) $h_{4}$

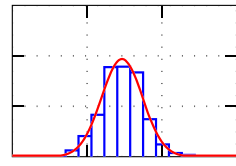

(f) $h_{6}$

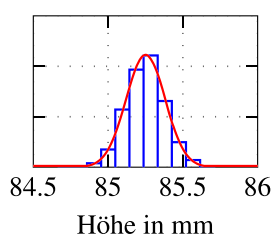

(h) $h_{8}$
Abb. 6. Verteilungsdichtefunktion der verschiedenen Messmethoden (a) $h_{1}$ bis (h) $h_{8}$ in Blau aus Abb. 5. In Rot ist die sich ergebende Normalverteilung gezeichnet. Die Messdauer beträgt $100 \mathrm{~s}$ (Farbabbildung online)

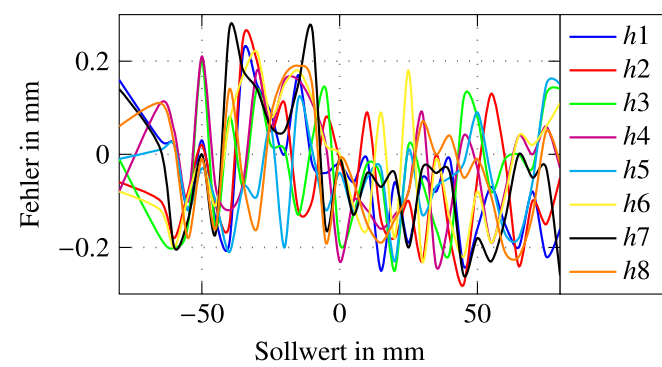

Abb. 7. Absoluter Fehler aller acht Messmethoden $\left(\boldsymbol{h}_{\mathbf{1}}\right.$ bis $\left.\boldsymbol{h}_{\mathbf{8}}\right)$ des Sensors über den Bereich von $\mathbf{- 8 0} \mathbf{m m}$ bis $\mathbf{1} \mathbf{8 0} \mathbf{m m}$. Es wurde alle $5 \mathrm{~mm}$ ein Messpunkt aufgenommen und die Differenz zum Sollwert berechnet. Die Messpunkte bei -75 mm, -70 mm und - $65 \mathrm{~mm}$ können nicht aufgenommen werden, da bei der verwendeten Montage des Sensors auf der Linearachse die Photodioden in diesem Bereich vom Montagewinkel für den Empfänger der Datenübertragung aus Abb. 4 abgedeckt sind. Bei einer anderen (verdrehten) Montage kann der nicht beleuchtete Bereich an den Rand des Messbereichs verschoben werden

Messmethode $h_{1}$ bis $h_{8}$ innerhalb des Bereichs von $\pm 0,3 \mathrm{~mm}$ bleibt. Bei dieser Messung wurden keine Mittelwerte gebildet. Dieser Wert kann durch einen gleitenden Mittelwert über mehrere Messungen bei geringen Bandbreitenanforderungen an den Sensor noch reduziert werden. 


\section{Zusammenfassung und Ausblick}

Zusammenfassend konnte gezeigt werden, dass das vorgestellte Sensorsystem in der Lage ist die Höhe und den Winkel in Bezug auf eine Laserreferenz berührungslos über eine Distanz von mehreren Metern mit einer Messgenauigkeit von $\pm 0,3 \mathrm{~mm}$ zu messen. Die auf Baustellen verwendeten Laser, die die Höhenreferenz in Bezug auf den Meterriss weitergeben, können als Referenz für den Sensor herangezogen werden, es muss nur die Modulierung zur Störungserkennung nachgerüstet werden. Mit diesem Messsystem ist es möglich, auf einem mobilen Verlegesystem für Estriche und Dämmschüttungen die Höhe und den Winkel in Bezug auf den "Meterriss"zu ermitteln.

Das Sensorprinzip wurde beim österreichischen Patentamt unter der Nummer A50253/2017 zum Patent angemeldet.

\section{Danksagung}

Open access funding provided by TU Wien (TUW). Diese Arbeit wurde durch die Österreichische Forschungsförderungsgesellschaft mbH (FFG) und das Land Oberösterreich im Rahmen des Basisprogramms unter der Antragsnummer 859241 finanziell unterstützt.

Open Access This article is distributed under the terms of the Creative Commons Attribution 4.0 International License (http://creativecommons.org/

\section{Autoren}

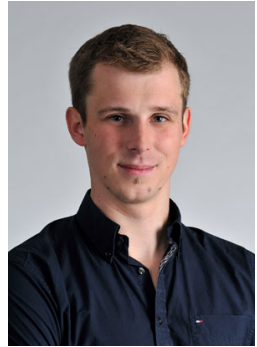

\section{Matthias Bib}

ist Forschungsassistent in der Advanced Mechatronic Systems-Gruppe am Institut für Automatisierungs- und Regelungstechnik (ACIN) der TU Wien, Österreich. Er absolvierte das Studium der Elektrotechnik an der TU Wien mit Auszeichnung (2014). Seine primären Forschungsinteressen sind Automatisierungssysteme, Messsysteme und Präzisionstechnik.

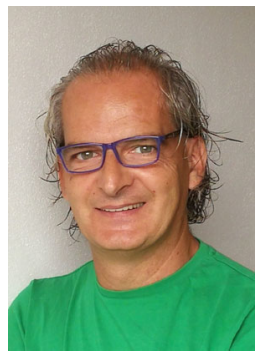

\section{Robert Stein}

ist interdisziplinärer Autodidakt der Ingenieurwissenschaften und beschäftigt sich seit mehr als 40 Jahren intensiv mit Elektronik, Mechanik, Elektrik und hat auch beruflich in verschiedenen Branchen immer damit zu tun.

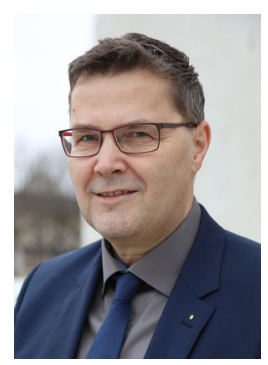

\section{Egon Döberl}

ist gelernter Maurer, der sich ständig weitergebildet hat, um seine Ideen verwirklichen zu können. Heute leitet er seit mittlerweile 15 Jahren erfolgreich seine Firmengruppe. Egon Döberl ist nicht nur als Geschäftsführer, sondern auch maßgeblich in den Bereichen Forschung und Entwicklung tätig. Sein Ziel ist es, Abläufe zu vereinfachen und zu perfektionieren und gleichzeitig die Arbeitsbedingungen seiner Mitarbeiter zu verbessern. licenses/by/4.0/), which permits unrestricted use, distribution, and reproduction in any medium, provided you give appropriate credit to the original author(s) and the source, provide a link to the Creative Commons license, and indicate if changes were made.

\section{Literatur}

1. Bauer, E. (2013): Energieeffizienz und Wirtschaftlichkeit. Tech. rep., Österreichischer Verband gemeinnütziger Bauvereinigungen - Revisionsverband.

2. Bauer, M., Hausladen, G., Hegger, M., Hegner, H. D., Lützkendorf, T., Radermacher, J., Sedlbauer, K., Sobek, W. (2011): Nachhaltiges Bauen (DIN Deutsches Institut für Normung e. V.

3. Zöller, A., Schossig, P., Haussmann, T. (2008): Wassergestützte latentwärmespeicher in putz- und dünnestrichsystemen. Tech. rep. Maxit Deutschland $\mathrm{GmbH}$, Fraunhofer Institut für Solare Energiesysteme

4. Schuth, M., Buerakov, W. (2017): Handbuch optische Messtechnik: praktische Anwendungen für Entwicklung, Versuch, Fertigung und Qualitätssicherung. München: Hanser.

5. Leica Geosystems (2013): Leica Rugby, 810, 820, 830 und 840.

6. Munnig Schmidt, R., Schitter, G., van Eijk, J. (2011): High performance mechatronics. Delft: Delft University Press.

7. Hilti Corporation (2015): PRA 38 Laserempfänger.

8. Vishay Semiconductors (2012): VSOP584

9. Dentan, M., de Cremoux, B. (1990): Numerical simulation of the nonlinear response of a $\mathrm{p}-\mathrm{i}-\mathrm{n}$ photodiode under high illumination. J. Lightwave Technol., 8(8), 1137.

10. STMicroelectronics (2013): STM32F103xB.

11. Schrüfer, E., Reindl, L., Zagar, B. (2012): Elektrische Messtechnik. Munich: Hanser Verlag.

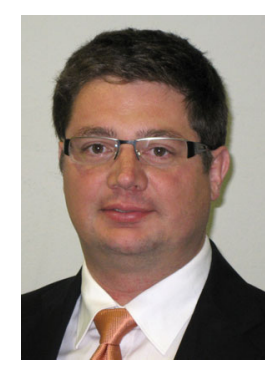

\section{Georg Schitter}

ist Professor für Advanced Mechatronic Systems am Institut für Automatisierungs- und Regelungstechnik (ACIN) der TU Wien, Österreich. Er absolvierte das Studium Elektrotechnik an der TU Graz, Österreich (2000) sowie ein Diplom in Informationstechnik und das Doktoratsstudium an der ETH Zürich, Schweiz (2004). Nach mehrjährigen Forschungsaufenthalten an der UCSB, USA, und TU Delft, Niederlande wurde er 2010 an die TU Wien berufen. Seine primären Forschungsinteressen befassen sich mit der Entwicklung integrierter Systemlösungen mechatronischer Systeme, inklusive neuer system- und regelungstechnischer Methoden, vor allem für Anwendungen in der Hochtechnologie, wissenschaftlicher Instrumentierung und bildgebende mechatronische Systeme, wie AFM, scanning Laser und LIDAR-Systeme, Teleskopsysteme, Adaptive Optik, und Lithographiesysteme für die Halbleiterindustrie. Für seine Forschung wurden Schitter einige Stipendien und Preise zugesprochen, darunter Stipendien des FWF und der Österreichischen Akademie der Wissenschaften sowie Best-Paper-Awards des IFAC Journal Mechatronics und des Asian Journal of Control. Schitter war Associate Editor der IFAC Journals Control Engineering Practice und Mechatronics sowie des Conference Editorial Boards der IEEE Control Systems Society (CSS) und der IEEE Transactions on Mechatronics. 